\title{
Latent image diffraction from submicron photoresist gratings
}

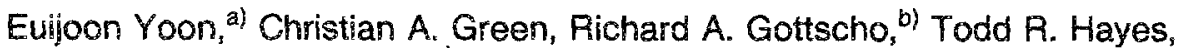 \\ and Konstantinos P. Giapis ${ }^{c)}$ \\ AT\&T Bell Laboratories. Murray Hill, New Jersey 07974
}

(Received 6 April 1992; accepted 17 June 1992)

\begin{abstract}
Light scattering from latent images in photoresist is useful for lithographic tool characterization, process monitoring, and process control. In particular, closed-loop control of lithographic processes is critical for high yield, low cost device manufacturing. In this work, we report use of pulsed laser diffraction from photoresist latent images in $0.24 \mu \mathrm{m}$ pitch distributed feedback laser gratings. Gated detection of pulsed light scattering permits high spatial resolution probing using ultraviolet light without altering the latent image. A correlation between latent image and etched grating diffraction efficiencies is demonstrated and shows the value of "upstream" monitoring.
\end{abstract}

As device dimensions continue to shrink, the complexity of microelectronic and photonic device processing increases and the need for better process monitoring and control becomes more critical. It is particularly important to develop methods for monitoring processes early in the device fabrication sequence. Such "up-stream" diagnostics are needed to avoid processing of material that is already defective. It is also important to monitor each process in the fabrication sequence to understand where additional control is needed: this approach leads directiy to higher precision manufacturing.

Lithography is a critical step used repeatedly in any device manufacturing sequence. Today, submicron lithographic exposures are "optimized" by using test wafers that are sent ahead of device wafers, exposed, developed, cleaved, and inspected using scanning electron microscopy (SEM). This approach is time-consuming, destructive, and "open-loop," i.e., without direct feedback for real-time exposure control. Open-ioop control cannot compensate for variations in exposure conditions that result from, for example, variable photoresist film thicknesses or fluctuations in light source intensity. As such, critical dimensions cannot be as precisely controlled as in a real-time, "closedloop" control system.

Recently, the use of light scattering to monitor microstructure for very large scale integrated (VLSI) circuit fabrication has been reported. ${ }^{1-10}$ Kleinknecht and Meier first used this technique to monitor linewidth and depth changes during wet and dry etching of $\mathrm{SiO}_{2}$ and $\mathrm{Si}_{3} \mathrm{~N}_{4}$ structures on Si. 'They showed that monitoring the first several diffraction orders from grating test patterns could be used for end-point detection. Grimard et al. showed that diffraction from isolated structures could be used to distinguish between relatively large $(20 \times 20 \mu \mathrm{m})$ rectangular and trapezoidal structures. ${ }^{8}$ Giapis et al. measured the scattered light intensity from large pitch grating test patterns and showed how this correlated with sidewall angle, etched depth, and surface composition. ${ }^{9}$ Naqvi et al. have developed a theoretical formalism that enables prediction of linewidths on photomasks by measuring the diffracted light intensity in transmission. ${ }^{10}$ In each of these cases, the structures examined were etched.

The basis for latent image monitoring, on the other hand, is the phase shift resulting simply from a difference in optical properties (index of refraction or absorption coefficient) between exposed and unexposed areas of the resist. ${ }^{11-14}$ Hickman et al. used diffraction of a HeNe laser beam from latent grating images to detect linewidth changes with exposure; they correlated diffraction from latent images with microstructure after development using SEM and simulated latent image diffraction using coupled wave analysis. ${ }^{11,13,14}$ Light scattering from latent images has also been used to characterize photolithographic exposure tools and conditions. ${ }^{11,12}$ The depth-of-focus, astigmatism, coma, and tilt of various toois can be determined from the intensity of light scattered from microstructure into a small solid angle. In this work, we apply latent image monitoring to the fabrication of submicron diffraction gratings made by coherent holographic lithography.

For any of these light scattering techniques, the ultimate sensitivity to small changes in linewidth, line profile, etched depth, etc. will be determined by the wavelength of the light used and the solid angle over which the light is detected. For example, consider the following scattering from a latent image diffraction grating:

$$
\sin \theta_{s}-\sin \theta_{i}=m \lambda / d,
$$

where $\theta_{s}$ and $\theta_{i}$ are the scattered and incident angles, respectively, $m$ is the diffraction order, $\lambda$ is the wavelength of the light, and $d$ is the grating period. From the relative intensities of the diffracted orders, the linewidth, line profile, and feature depth can be determined. ${ }^{11,13,14}$ However, from $\mathrm{Eq}$. (1) we see that the number of diffracted orders is determined by the ratio of the grating pitch to the wavelength of the light; therefore, the precision with which microstructural properties can be determined is limited by the number of diffracted orders that can be detected. The shorter the wavelength, the larger the number of orders. Depending on the wavelength dependence of the optical properties of exposed and unexposed resist as well as un- 


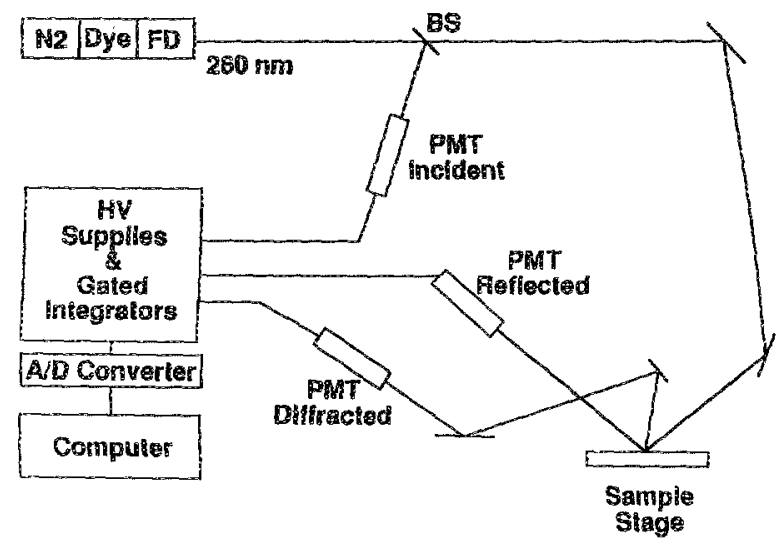

FIG. 1. Schematic illustration of pulsed laser latent image monitoring experiment.

derlying materials, shorter wavelength light may also be needed to obtain adequate sensitivity.

Unfortunately, shorter wavelength light is also absorbed more strongly by photoresists. To use shorter wavelengths and not alter the latent image, the probing radiation dose must be sufficiently low. For example, if we require an exposure dose of $0.01 \mathrm{~J} / \mathrm{cm}^{2}$, then a probe dose of no more than $10^{-5} \mathrm{~J} / \mathrm{cm}^{2}$ would provide adequate assurance that the probe is nonperturbative. Such energy densities are easily obtained in the ultraviolet by frequency doubling pulsed lasers. With fast pulse probing, gated detection can be used to discriminate against room light, photoresist fluorescence, and dark current. The monochromatic and low divergence properties of laser light are also useful for reducing room light and fuorescence interference.

In this work, we used a pulsed $\mathrm{N}_{2}$ laser to pump a frequency-doubled, tunable dye laser, and monitor latent image diffraction from $0.24 \mu \mathrm{m}$ pitch gratings in photoresist used in the fabrication of distributed feedback (DFB) lasers. ${ }^{15-17}$ The laser is attenuated so that no more than $-10 \mathrm{~nJ} / \mathrm{cm}^{2}$ per pulse at $260 \mathrm{~nm}$ are used to scatter from the latent image. Although $\sim 98 \%$ of the laser light is absorbed at this waveiength, this intensity is sufficiently small to avoid distortion of latent images and yet large enough to provide good signal to noise ratio in detecting the first order and specular peaks. In this case diffraction may result not only from differences in index of refraction between exposed and unexposed regions but also from differences in absorption.

Incident, specular, and first order diffracted beams are detected using solar blind photomultiplier tubes, PMTs (Hamamatsu R431S) (Fig. 1). Color glass bandpass $(248-390 \mathrm{~nm}$, Oriel no. 59800) filters are also used to help discriminate against room light and photoresist fuorescence. The incident and specular beams are further attenuated by 100 times to avoid PMT saturation.

Holographic gratings are fabricated by interfering two beams from a single-transverse-mode, muitilongitudinalmode $325 \mathrm{~nm} \mathrm{cw}$ HeCd laser in $40 \mathrm{~nm}$ Shipley PR 1400-3 photoresist on InP substrates. ${ }^{16}$ Exposures take typically $50 \mathrm{~s}$ for a total dose of $\sim 8 \mathrm{~mJ} / \mathrm{cm}^{2}$. The latent images are not developed before being transferred in a light-tight con-
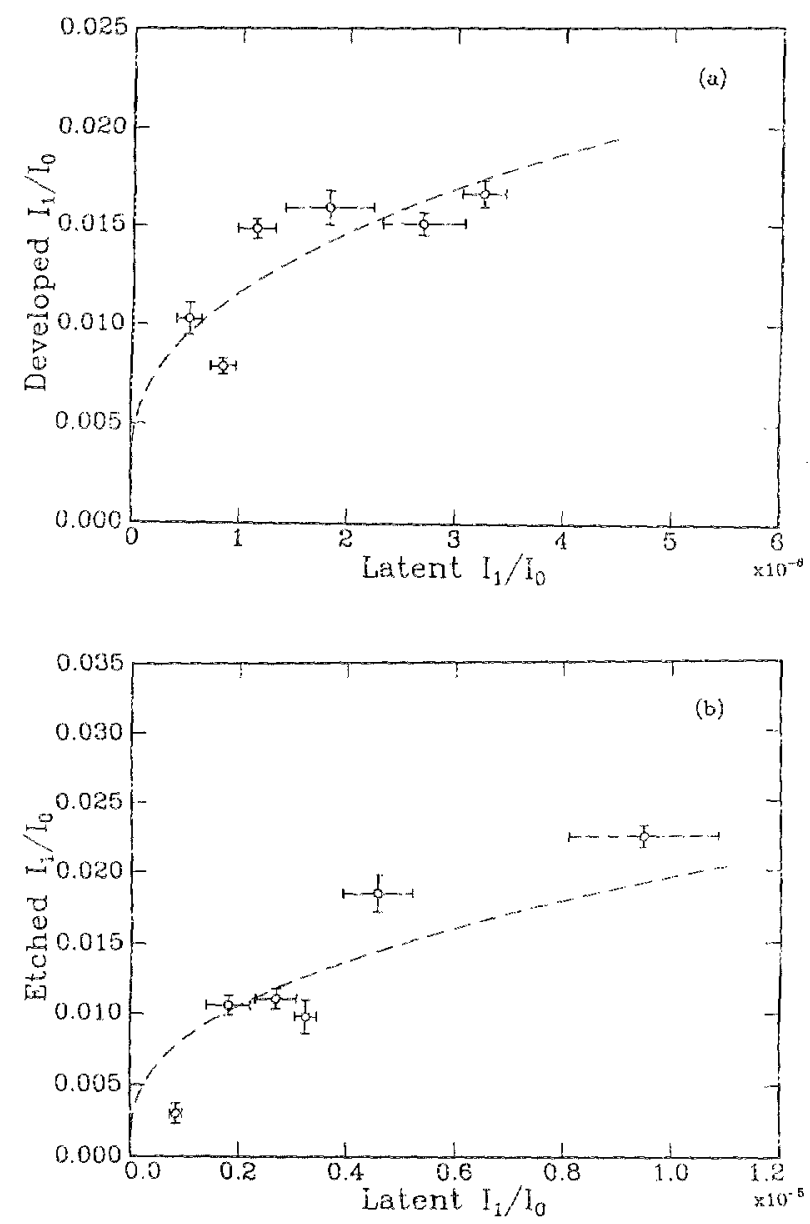

Fig. 2. (a) Developed grating diffraction efficiency (diffracted/incident intensity ratio) vs latent image diffraction efficiency. (b) Etched grating diffraction efficiency vs latent image diffraction efficiency.

tainer to another laboratory where the pulsed laser diffraction experiments are performed. Wafers are mounted vertically onto a vacuum chuck after the system has been prealigned using etched gratings. The wafer is exposed briefly to the laser beam to optimize the signal and then translated without affecting the alignment to measure diffraction efficiency at different locations on the wafer surface. The laser spot size on the wafer is approximately 0.5 $\mathrm{cm} \times 1.0 \mathrm{~cm}$ but could be made much smaller by using a lens to focus the beam. The laser beam is shuttered such that each spot is only exposed to 10 pulses for a dose of $\leqslant 10^{-7} \mathrm{~J} / \mathrm{cm}^{2}$, which is much less than would be needed to aiter the latent image. Even after a continuous $13 \mathrm{~min}$ exposure at $20 \mathrm{~Hz}$ for a dose of $\sim 10^{-4} \mathrm{~J} / \mathrm{cm}^{2}$, the diffraetion efficiency does not change indicating that the latent image has not been perturbed. By tuning the laser to even shorter wavelengths, $<0.24 \mu \mathrm{m}$, a second order diffraction peak could be detected to gain further information on latent image properties without risking latent image distortion.

After the latent image diffraction measurements are made, the photoresist is developed and diffraction effciency is again recorded. To transfer the grating into the InP substrate, wafers are reactively ion etched to a depth of 
$80 \mathrm{~nm}$ using a $\mathrm{CH}_{4} / \mathrm{H}_{2}$ plasma. ${ }^{17}$. The photoresist is then stripped and the diffraction efficiency measured a third time. Finally, wafers are inspected using a SEM.

Correlations between diffraction efficiencies from latent image, developed grating, and etched grating are shown in Fig 2. Note that the correlations are generally not expected to be linear, since the diffraction efficiency as a function of duty cycle (or exposure time) may have a different func-

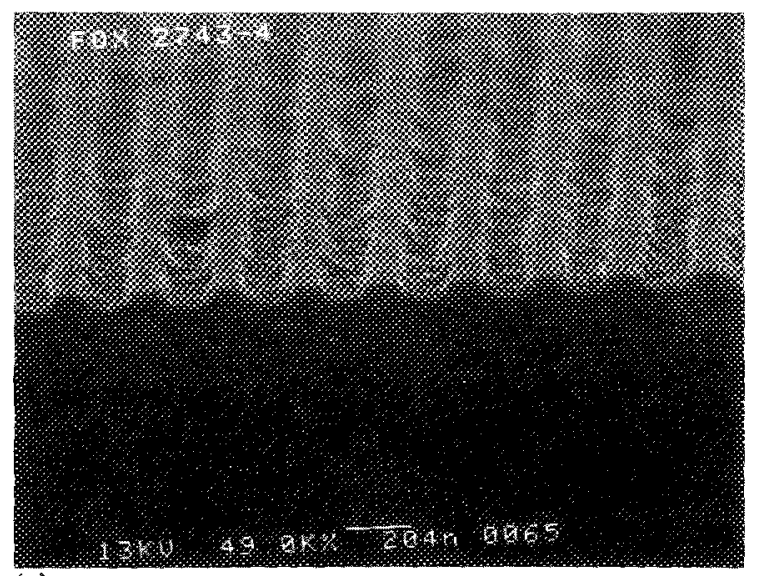

(a)

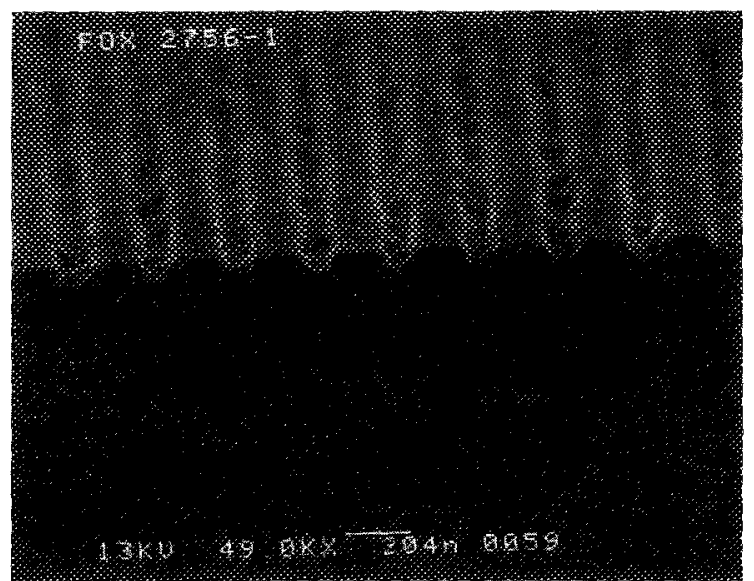

(b)

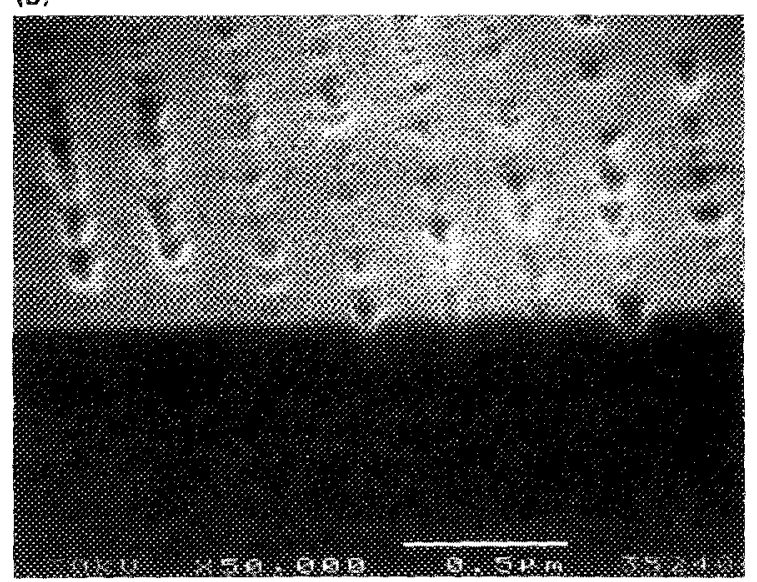

(c)

Frg. 3. Scanning electron micrographs of gratings etched in $\operatorname{In} P$ after coherent holographic exposure and development. Although each wafer was processed in the same manner, the resultant gratings are clearly different and exhibit (a) high, (b) intermediate, and (c) low latent image diffraction efficiency. tional dependence for each of the gratings-latent, developed, and etched. For example, when the latent image grating diffraction efficiency is a maximum, the diffraction efficiency of the developed and etched gratings could be either before or past their corresponding maxima. Thus, the correlations will generally be double-valued but must always pass through the $(0,0)$ point; the curves shown in Fig. 2 are intended to only help guide the eye. In any case, latent image diffraction intensity is an accurate predictor of both developed and etched grating diffraction efficiency.

The "error" bars shown in Fig. 2 represent real variations in diffraction efficiency with position on the wafer. Such variation could result from the short coherence length of the exposing laser radiation, variation in laser intensity, standing waves in the resist, scattered radiation, etc. The inherent reproducibility in the measurements at a given location is comparable to the size of the open circles used to represent the average efficiency data.

Each of the gratings shown in Fig. 3 received nominally the same dose and were developed for nominally the same time. Clearly, the run-to-run reproducibility is not very good. For three reasons we believe this variability stems from poor process control during exposure: (1) both developed and etched grating efficiencies show similar correlations with latent image diffraction efficiency (Fig. 2); (2) the $\mathrm{HeCd}$ laser used for exposure here was known to have mode instability problems; and, (3) since this work began, the laser has been replaced with another having superior amplitude and mode stability and the run-to-run reproducibility has been improved.

Regardless of the run-to-run reproducibility, the SEMs in Fig. 3 show that gratings with different diffraction efficiency exhibit different microstructure. The diffraction efficiencies of the gratings clearly correlate with duty cycle, i.e., the ratio of linewidth to grating pitch, but line edge roughness, or edge acuity, may also play a role. Poor edge acuity can result in diffuse scattered light intensity that is "stolen" from the coherently diffracted beam.

These results illustrate the power of latent image monitoring in isolating process steps that are not adequately controlled. At the very least, latent image monitoring can be used to signal catastrophic failure during the exposure. The next step will be to implement the method in real time and to exert closed-loop control on the exposure process.

${ }^{a}$ Current address: Seoul National University, Materials Science Department, Seoul, S. Korea.

b) Author to whom correspondence should be addressed.

${ }^{c)}$ Current address: Chemical Engineering Department, California Institute of Technology, Pasadena, CA.

${ }^{\prime}$ H. P. Kleinknecht and H. Meier, J. Electrochem. Soc. 125, 798 (1978).

${ }^{2}$ H. P. Kleinknecht and H. Meier, Appl. Opt. 19, 525 (1980).

${ }^{3}$ H. P. Kieinknecht, Inst. Phys. Conf. Ser. No. 69, 29 (1984).

${ }^{4}$ E. S. Braga, G. F. Mendes, J. Frejlich, and A. P. Mammana, Thin Solid Films 109, 363 (1983).

${ }^{5}$ G. F. Mendes, L. Cescato, and J. Frejlich, Appl. Opt. 23, 571 (1984).

${ }^{6}$ G. F. Mendes, L. Cescato, and J. Frejlich, Appl. Opt. 23, 576 (1984).

${ }^{7}$ G. F. Mendes, L. Cescato, J. Frejlich, E. S. Braga, and A. P. Mammana, Thin Solid Films 117, 107 (1984).

${ }^{8}$ D. S. Grimard, F. L. Terry, Jr, and M. E. Elta, Proc. SPIE 1185, 234 (1990).

${ }^{9}$ K. P. Giapis, R. A. Gottscho, L. A. Clark, J. B. Kruska1, D. Lambert, A. Kornblit, and D. Sinatore, J. Vac. Sci. Technol. A 9, 664 (1991). 
${ }^{10}$ S. S. H. Naqui, S. Gaspar, K. Hickman, K. Bishop, and J. R. McNeil, Appl. Opt. 31, 1377 (1992).

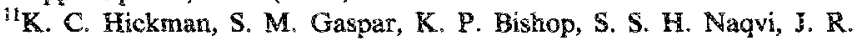
McNeil, G. D. Tipton, B. R. Stallard, and B. L. Draper, Proc. SPIE 1464, 245 (1991).

${ }^{12}$ T. E. Adants, Proc. SPIE 1464, 294 (1991).

${ }^{13}$ L. M. Milner, K. C. Hickman, S. M. Gaspar, K. P. Bishop, S. S. H. Naqvi, J. R. McNeil, M. Blain, and B. L. Draper, Proc. SPIE 1673, 274 (1992).

${ }^{14}$ K. C. Hickman, S. M. Gaspar, K. P. Bishop, S. S. H. Nagvi, J. R.
McNeil, G. D. Tipton, B. R. Staliard, and B. L. Draper, J. Vac. Sci. Technol. B 10, 2259 (1992).

${ }^{15}$ N. K. Dutta, Y. Twu, C. Y. Kuo, A. B. Piccirilli, and R. L. Brown, Proc. SPIE 992, 287 (1988).

${ }^{16}$ C. A. Green, A. B. Píccirilli, A. G. Lehrer, C. B. Roxlo, Y. Twu, and D. T. C. Huo (unpubiished results)

${ }^{17}$ T. R. Hayes, S. J. Kim, and C. A. Green, Proc. SPIE 1818, 190 (1991). T. R. Hayes, M. A. Dreisbach, P. M. Thomas, W. C. DautremontSmith, and L. A. Heimbrook, J. Vac. Sci. Technol. 8 7, 1130 (1989). 